\title{
Spinal cord toxoplasmosis as an unusual presentation of AIDS: case report and review of the literature
}

\author{
Carlos Garcia-Gubern • Carmen R. Fuentes • \\ Lissandra Colon-Rolon • Daniel Masvidal
}

Received: 2 October 2009 / Accepted: 15 June 2010 /Published online: 7 September 2010

(C) Springer-Verlag London Ltd 2010

\begin{abstract}
Approximately $10 \%$ of patients with AIDS present with some neurological deficit as their initial complaint, and up to $80 \%$ will have CNS involvement during the course of their disease. Toxoplasmosis is the most common cause of cerebral mass lesions in patients with AIDS, but appears to be an uncommon cause of spinal cord disease. The incidence of myelopathy may be as high as $20 \%$, with $50 \%$ of the cases reported postmortem. We present a unique case of spinal cord disease as the initial presentation of AIDS. We also present a comprehensive literature review of this topic, its diagnosis and treatment. This is a retrospective chart review case report. After a detailed case presentation, several diagnostic and therapeutic aspects of this unique case are thoroughly discussed. Although spinal cord toxoplasmosis is uncommon, it has been suggested that most patients with AIDS that present with evolving myelopathy, characterized by extremity weakness, sensory involvement, spinal cord enlargement, enhancing lesions in brain or spinal cord CT or MRI, have toxoplasmic myelitis.
\end{abstract}

Keywords AIDS · Spinal toxoplasmosis .

Emergency department

C. Garcia-Gubern $(\bowtie) \cdot$ C. R. Fuentes $\cdot$ L. Colon-Rolon •

D. Masvidal

Department of Emergency Medicine,

Hospital San Lucas/Ponce School of Medicine,

Ponce, Puerto Rico

e-mail: prerdoc@yahoo.com

\section{Introduction}

Although spinal cord abnormalities in patients with acquired immunodeficiency syndrome (AIDS) have been infrequently reported in the literature, myelitis is a known complication of AIDS and is occasionally the initial complaint. The incidence of myelopathy may be as high as $20 \%$, with $50 \%$ of the cases reported post-mortem [3, 5]. Toxoplasmosis is the most common cause of intracranial lesions responsible for neurological deficits in AIDS patients, occurring in $3-10 \%$ of patients in the United States and in up to $50 \%$ of AIDS patients in Europe, Latin America, and Africa [6, 10]. A review of existing literature suggests that although Toxoplasmic myelitis is uncommon, it should be suspected in immunocompromised patients who present with symptoms of acute or sub-acute myelopathy [7]. The initial evaluation should aid in differentiating between other reported causes of myelopathy (such as vacuolar myelopathy, lymphoma, tuberculosis, and viral infections including cytomegalovirus infection, herpes zoster, and herpes simplex) in AIDS patients [2, 4]. Since 1986, 18 cases of apparent toxoplasmosis of the spinal cord have been described $[4,7,11,12]$. A case report and pertinent literature were reviewed, leading to the diagnosis and management options discussed below.

\section{Case report}

A 40-year-old Hispanic man was admitted to the hospital after being found unconscious. He had a 2-day history of disorientation that manifested itself as his being unable to recognize family members. Upon admission he regained consciousness, becoming alert and oriented, but developed urinary retention and was unable to move or feel his lower extremities. He had no history of systemic illness, but 


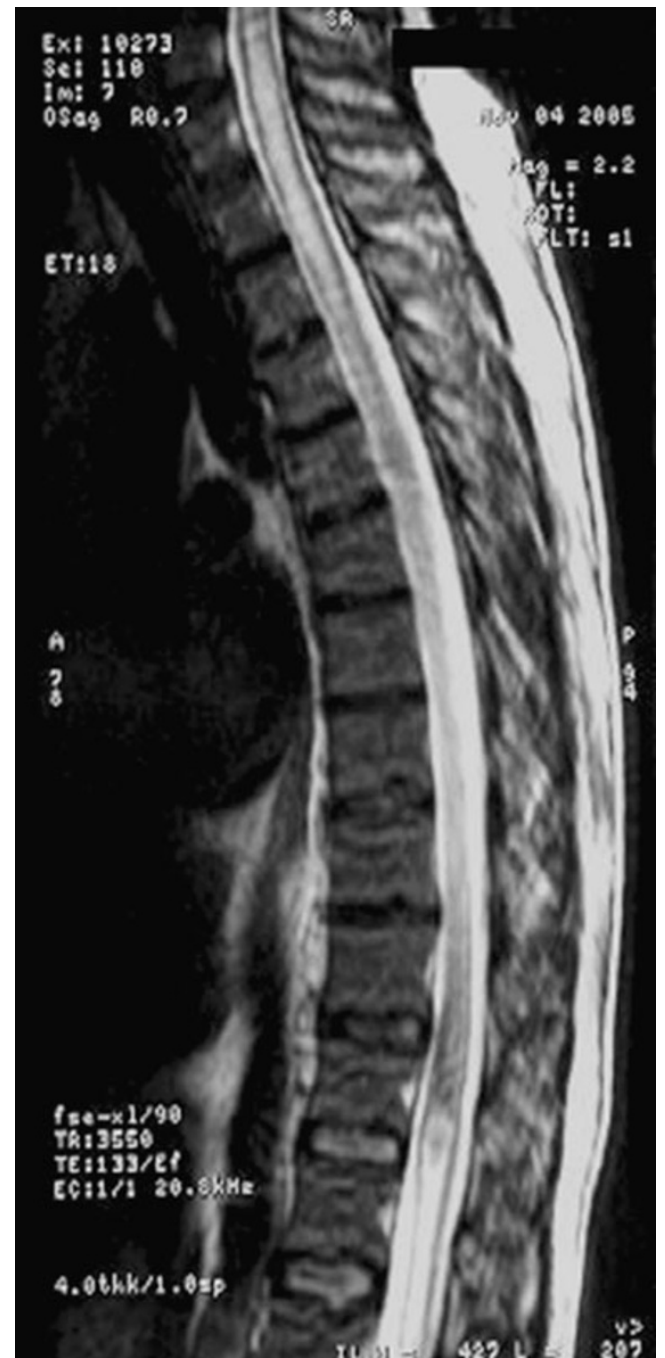

Fig. 1 Initial MRI: notice the abnormal spinal cord swelling cervical and at cauda equina

mentioned having been treated for herpes zoster approximately 3 years prior. He denied having a history of fever, chills, nausea, vomiting, rash, seizures, or other constitutional symptoms. He had not traveled recently, and his HIV status was unknown at the time of admission.

Physical examination revealed fever, nuchal rigidity, and a distended bladder that required catheterization. No rash, erythema, oral lesions, lymphadenopathy, or papilledema was noted. A neurological examination confirmed that he had intact cranial nerves and normal upper extremity strength. Both lower extremities exhibited flaccid paralysis and reduced response to pain, touch, and temperature (bilaterally from L1). There was impairment of propioception and vibration. Cerebellar examination was normal in the upper extremities, with an adequate finger-to-nose exam. The patient's WBC, hemoglobin, platelets, electrolytes, BUN, and creatinine were within normal ranges on admission. The initial brain CT (without contrast) revealed a focal area of vasogenic edema in the left frontal lobe (without significant mass effect) and abnormal white matter hypo-density seen along the lateral aspect of the right frontal horn of the internal capsule. A brain MRI (with and without gadolinium enhancement) revealed multiple bilateral ring-enhancing intra-axial brain lesions, and an ill-defined cortical and sub-cortical enhancement, with diffuse edema in the frontal lobe.

Spinal MRI (with and without gadolinium) showed the spinal cord to be abnormally diffuse, with swelling and edema in the cervicothoracic region. Along with the diffuse abnormal hyperintense swelling, the cauda equina had an edematous appearance, and signal intensity was abnormally increased, which is compatible with transverse myelitis (Figs. 1 and 2). Finally, imaging also revealed a focal area of abnormal signal intensity in the ventral inferior pons.

$\mathrm{He}$ was initially given broad spectrum antibiotics (ceftriaxone, vancomycin, and ampicillin) and acyclovir at admission for a presumptive CNS infection. HIV serology was recommended, though the patient denied ever having engaged in high-risk behavior or having had a blood transfusion at any time in his past. Analysis of CSF showed abnormal values including a WBC count of $11 / \mathrm{mm}^{3}$ and a protein level of $184.5 \mathrm{mg} / \mathrm{dl}$, with a glucose concentration

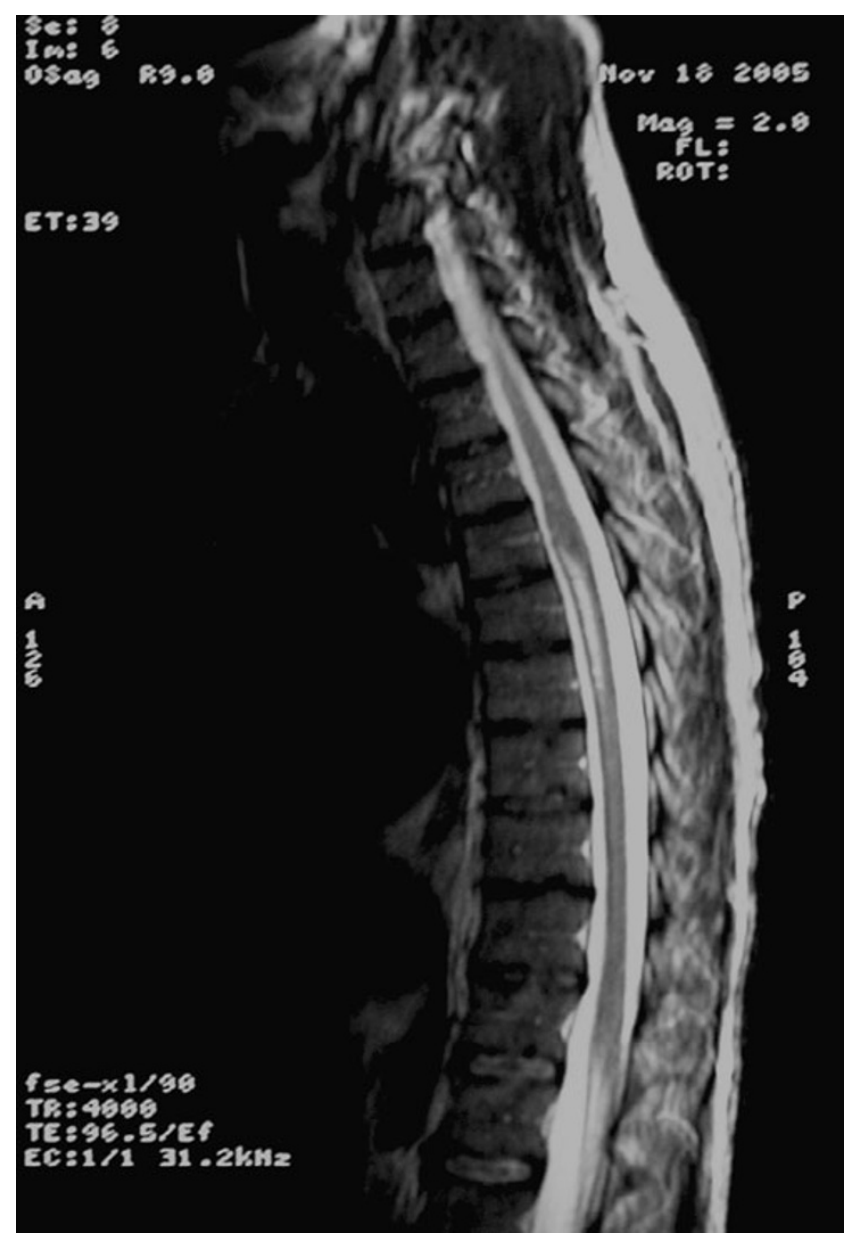

Fig. 2 Normal F/U study 
of $51 \mathrm{mg} / \mathrm{dl}$. Stain and cultures for bacteria, fungi, and acidfast bacilli were negative. A CSF cryptococcal antigen titer and HTLV I/II antibody assay were negative; a VDRL test was non-reactive. Cytology revealed pleocytosis with a predominance of mononuclear lymphoid-like cells. An antitoxoplasma immunoglobulin (IgG) immune titer was positive at $8.99(<0.9=$ negative; $\geq 1.10=$ positive $)$. At this point, the patient disclosed having engaged in homosexual behavior and accepted HIV testing, which was reactive by ELISA. Empiric treatment for toxoplasmosis with sulfadiazine, pyrimethamine, folinic acid, and HAART therapy with lamivudine/zidovudine and indinavir were started. Intravenous dexamethasone was administered for 7 days, and oral methylprednisolone for another week. A lymphocyte subset panel revealed a CD4 count of 56 cells $/ \mathrm{mm}^{2}$. Positive Western blot results arrived, confirming a diagnosis of HIV. Neither PET nor SPECT was available for an additional imaging workup. Biopsy was deferred due to possible complications and the evidence of improvement with therapy.

Within 4 days of empiric treatment for toxoplasmosis, the patient's neck rigidity resolved. He progressively developed discrimination to touch and his vibratory sensation improved, as did his bilateral strength (1/5) of the lower extremities. Follow-up consisted of a brain and spinal cord MRI (with and without gadolinium), 14 days after treatment with anti-toxoplasmosis therapy combined with steroids, which demonstrated resolved swelling and residual T2 hyper-intensity in the mid-thoracic spinal cord, without an interval change in the brain MRI. The patient was discharged home with rehabilitation therapy after 22 days of admission.

\section{Discussion}

AIDS-related spinal cord disorders include neoplasms, infections (including HIV itself), vascular disease, and other undefined etiologies. Toxoplasmosis and lymphoma are the two most common intracranial lesions, and both have been reported in increasing frequency in the spinal cord [1]. Myelopathy is usually under-diagnosed, probably because of the occurrence of coexisting conditions such as AIDS dementia complex (ADC), cerebral lesions of varying etiologies, vacuolar myelopathy, lumbosacral myelopathy, or peripheral neuropathy that may mask the clinical signs suggestive of myelopathy [5, 9, 10]. Other causes of myelopathy appear to be less common than toxoplasmosis and include tuberculoma, cytomegalovirus, varicella-zoster virus, and, possibly, lymphoma [4, 10]. Postmortem histopathology examination has resulted in a definitive diagnosis of myelopathy in the majority of patients [7].

Spinal cord lesions often manifest with a variety of symptoms, such as leg weakness, progressive paraparesis with spasticity, absent reflexes, ataxia, incontinence, and paresthesias [13]. In the 14 toxoplasmosis cases with spinal cord involvement reviewed by Vyas and Ebright, they found the most common presentations of acute or sub-acute symptoms were paraparesis, urine retention, sensory level deficits, fever, and local pain. CSF cytology showed increased protein-level elevation and a CD4 count of less than $50 / \mathrm{mm}^{3}$. In the patients evaluated by spinal cord MRI imaging, localized intramedullary lesions or spinal cord edema were found in more than $90 \%$ of cases associated with positive $T$. gondii $\operatorname{IgG}$ antibody [3]. No cases of associated transverse myelitis, as in this case, were described. In our case, diagnosis was based on the clinical picture and response to therapy.

Evaluation in a symptomatic patient should include serum and CSF cytology and antibody (immunological) studies as they remain the gold standard for identifying an infectious agent. Complete radiographic imaging of the entire neuroaxis $[3,15]$ is key in clearly defining inflammatory lesions of the brain and spine, and in the visualization of typical lesions that allow for rapid diagnosis. This helps determine whether the lesion is extramedullary or an intrinsic spinal cord disease, which in turn provides information to determine whether surgical or medical therapy is needed [3]. In opportunistic diseases, the imaging studies also have a crucial role in diagnosis and monitoring of the therapeutic response [8].

Neuroimaging findings, along with other clues, help to narrow the differential diagnosis. On an MRI, findings of a normal-sized spinal cord but with an abnormal signal should signal the possibility of vacuolar myelopathy and ADC-related HIV myelitis. Lesions of viral myelitis can potentially cause spinal cord enlargement; however, laboratory data are required for confirmation [3]. If spinal cord enlargement is present, toxoplasma myelitis and lymphoma should be strongly considered [14]. Lymphoma has been associated with positive CSF polymerase chain reaction (PCR) for Epstein-Barr virus [9]. If both spinal cord enlargement and an abnormal signal are associated with meningeal enhancement, then CMV, $M$. tuberculosis, lymphoma, and toxoplasmosis should be considered along with other less likely infectious causes [3].

MRI (plain and contrast-enhanced) is currently considered appropriately sensitive for detecting brain and spinal cord lesions; however, equal sensitivity has been reported using delayed-contrast CT scanning [1]. The current guidelines for diagnosis of intracranial lesions state that T1 SPECT is an option when available. When positive (marked uptake in contrast to toxoplasma), it appears to be highly specific for primary CNS lymphoma [6, 8]. These guidelines have been described as helpful [3] in spinal cord lesions and suspected lymphoma, although specific guidelines for spinal cord lesions are not presently available. The newer and more available MRI techniques of diffusion-weighted imaging (DWI) may be of help in the differentiation of lymphoma, showing no restriction in water diffusion [8]. If a diagnosis of solitary or atypical lesions cannot be made with noninvasive methods, a 
biopsy (open or stereotactic) may be warranted in patients with concomitant negative toxoplasmosis serology since a negative serology does not exclude diagnosis of toxoplasmosis nor differentiate from lymphoma. A biopsy may also be considered necessary if a patient experiences a rapid decline in function or, alternately, fails to improve despite therapy [1].

Brain biopsies have been associated with hemorrhage risk and an increased mortality $(2 \%)$ and morbidity rate $(12 \%)$ in patients with HIV/AIDS. The estimated rate of non-diagnostic brain biopsies ranges between 4-36\% [6].

Empiric treatment for toxoplasmosis with oral pyrimethamine and sulfadiazine (with folinic acid) has been recommended in all cases of intracranial mass lesions in patients with HIV/AIDS (except in a solitary mass with negative toxoplasma serology) [6]. It is also believed to be beneficial when there is spinal cord involvement [2]. Steroids promote radiological improvement in about $80 \%$ of patients, and improvement can be seen in about 1 week, supporting the diagnosis [8]. Patients are usually monitored clinically and radiographically for response to treatment over a 10- to 14-day period following empiric therapy. If responsive, anti-toxoplasmosis therapy is continued indefinitely, and reevaluation in the absence of steroid treatment is mandated. If the lesions remain unchanged or progress, the diagnosis has to be reconsidered and the therapeutic strategy reevaluated. In cases of atypical large solitary toxoplasma lesions resembling lymphoma (that also present with spinal cord enlargement), additional diagnostic modalities should be performed $[3,6]$. Due to the immunosuppressant effects of steroids [9], the optimal dosage and period of treatment for spinal cord lesions must be addressed in future studies.

In this case, the brain and spinal cord lesions were found in an HIV-positive man who had not previously been diagnosed. For this reason, other causes of CNS infection and lymphoma were first considered. After the positive serology, empiric treatment for cerebral toxoplasmosis was initiated. The patient's clinical and radiographic improvement led to the final diagnosis of toxoplasmic myelitis and encephalitis, similar to other cases documented in the literature by Vyas et al. in 1996 [4].

Acute myelopathy was one of the first manifestations of AIDS in this patient. Many patients with neurological disease are unaware of their HIV status. Furthermore, toxoplasmic myelopathy has been described in patients without HIV, albeit with severe immunosuppression [2, 3, 11, 12], and in patients with isolated spinal cord lesions $[1,2,11]$. New neurological deficit in any patient should raise a high index of suspicion of HIV infection. Appropriate diagnostic methods and management that are practical for all settings, including those with limited technologies, should be sought. Newer methods of diagnosis and management for all neurological complications of HIV should be addressed. An algorithm for the evaluation and management of spinal cord lesions should be established [4]. We established ours based on the clinical manifestations, history, and general improvement of the patient's condition.

Although spinal cord toxoplasmosis is uncommon, it has been suggested that most patients with AIDS that present with evolving myelopathy, characterized by extremity weakness, sensory involvement, spinal cord enlargement, and enhancing lesions in brain or spinal cord CT or MRI, have toxoplasmic myelitis [3,5]. The likelihood of diagnosis increases if serum titers for Toxoplasma antibodies are positive or where the initiation of early treatment with empiric anti-toxoplasmosis therapy and steroids improves both the patient's clinical and radiographic manifestations.

Conflicts of interest None.

\section{References}

1. Daniel R, Christopher C, William W, Julio M, William H, George J (1995) Isolated toxoplasmosis of the thoracic spinal cord in a patient with acquired immunodeficiency syndrome. J Neurosurg 82:493-496

2. Nag S, Jackson AC (1989) Myelopathy: an unusual presentation of toxoplasmosis. Can J Neurol Sci 16:422-425

3. Quencer RM, Judith D (1997) Spinal cord lesions in patients with AIDS. Neuroimaging Clin N Am 7(2):359-373

4. Rohit V, John E (1996) toxoplasmosis of the spinal cord in a patient with AIDS: case report and review. Clin Infect Dis 23:1061-1065

5. Herskovitz S, Siegel SE, Schneider AT, Nelson SJ, Goodrich JT, Lantos G (1989) Spinal cord toxoplasmosis in AIDS. Neurology 39:1552-1553

6. Gildenberg PL (1998) Evaluation and management of intracranial mass lesions in AIDS: Report of the Quality Standards Subcommittee of the American Academy of Neurology. Neurology 50:21-26

7. Campo C, Navarro V, Mota JA, Lacruz J, Santos M (2001) Spinal cord lesion in a patient with human immunodeficiency virus infection. Enferm Infecc Microbiol Clín 19(1):31-33

8. Oliver K, Isabel W, Matthias M (2005) Neuroimaging of infections. NeuroRx 2(2):324-332

9. Todd GJ, Estil V, Peter H, Tanner M (2000) Brain lesion and AIDS. Proc Bayl Univ Med Cen 13(4):424-429

10. Vijay T, Sudha T, Lakshmi V (2005) Neurologic manifestations of HIV infection: An Indian hospital-based study. AIDS Read 15(3):139-145

11. Straathof CS, Kortbeek LM, Roerdink H, Sillevis Smitt PA, van den Bent MJ (2001) A solitary spinal cord toxoplasma lesion after peripheral stem-cell transplantation. J Neurol 248(9):814-815

12. Cosan TE, Kabukcuoglu S, Arslantas A, Atasoy MA, Dogan N, Ozgunes I, Kebabci M, Tel E (2001) Spinal toxoplasmic arachnoiditis associated with osteoid formation: a rare presentation of toxoplasmosis. Spine 26(15):1726-1728

13. Bowen J, Acute viral infections of the spinal cord. www.myelitis. org/baltimore2001/bowen_handout.pdf

14. Manelfe C (1992) Imaging of tumoral and non tumoral diseases of the spinal cord. In: Imaging of the spinal cord, vol. 1. Raven Press, NY. Found at www.starrprogram.com/upload/starabstracts 6842001

15. HIV Guidelines (2004) New York State Department of Health AIDS Institute: Neurologic complications. www.hivguidelines. org/public_html/a-neuro/a-neuro.htm. March 